\title{
EFFECT OF CEFOVECIN ON THE FECAL FLORA OF HEALTHY DOGS
}

\author{
by
}

MEGAN RENE LAWRENCE

B.A., Kansas State University, 2012

\begin{abstract}
A REPORT
submitted in partial fulfillment of the requirements for the degree
\end{abstract}

\section{MASTER OF SCIENCE}
Department of Biomedical Sciences
College of Veterinary Medicine

\section{KANSAS STATE UNIVERSITY \\ Manhattan, Kansas}

2014

Approved by:

Major Professor

Dr. Sanjeev K. Narayanan 


\begin{abstract}
Cefovecin is an extended-spectrum long-acting third generation cephalosporin used to treat canine infections. The study objective was to determine the effect of cefovecin on the absolute number and antimicrobial susceptibility of fecal enteric bacteria in healthy dogs. Fourteen Beagles were randomly assigned to a treated $(n=7,8 \mathrm{mg} / \mathrm{kg}$ cefovecin subcutaneously on day 1$)$ or untreated $(n=7)$ group. LC/MS was used to determine plasma cefovecin concentration on day 14. E. coli, enterococci, and Salmonella were isolated and enumerated from fecal samples collected on days $0,3,7,14$, and 28. Antimicrobial resistance was determined using disc diffusion, MIC, and detected using PCR for the $b l a_{\mathrm{CMY}-2}$ gene on select isolates. Mean plasma concentration of cefovecin on day 14 was $9.59 \mu \mathrm{g} / \mathrm{mL}$ in treated dogs; untreated dogs had no measurable plasma cefovecin. The absolute number of E. coli was lower in treated dogs on day $3(P \leq 0.0001)$, and the absolute number of cefovecin-resistant $E$. coli was higher in treated dogs on days $7(P=0.002), 14(P=0.004)$ and $28(P \leq 0.0001)$, compared to untreated dogs. Enterococci increased and were higher in the treatment group on day 7 ( $P=$ 0.0226). Isolation of Salmonella was rare. After cefovecin treatment, beta-lactam resistance was more common in fecal E. coli from treated dogs than untreated dogs, while resistance of enterococci was not altered. On day 28, treated dogs were 3.25 times more likely to carry the bla $a_{\mathrm{CMY}-2}$ gene than untreated dogs $(95 \%$ CI $1.27-8.35)$. The implications of these findings in clinically ill patients require further research.
\end{abstract}




\section{Table of Contents}

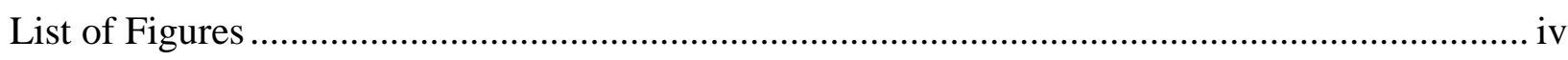

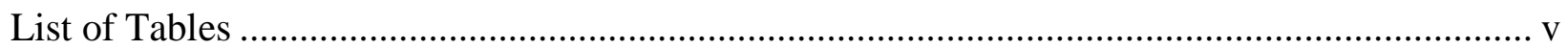

Acknowledgements ........................................................................................................... vi

Chapter 1 - Introduction ............................................................................................... 1

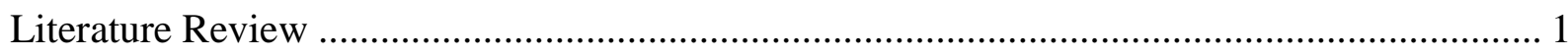

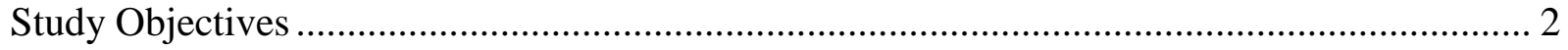

Chapter 2 - Materials and Methods....................................................................................... 3

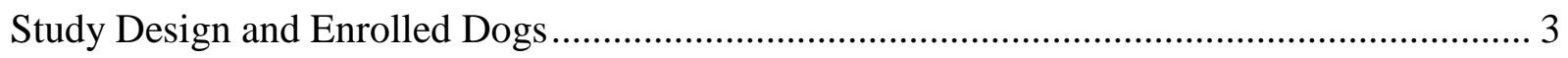

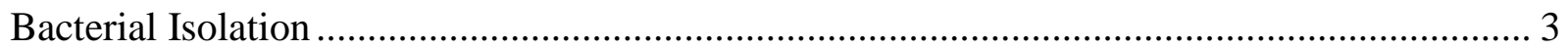

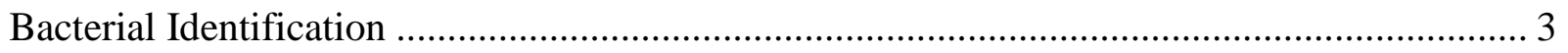

Plasma Cefovecin Concentration Analysis ........................................................................ 4

Antimicrobial Susceptibility Testing .................................................................................... 4

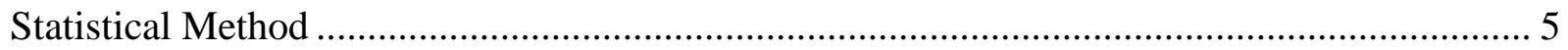

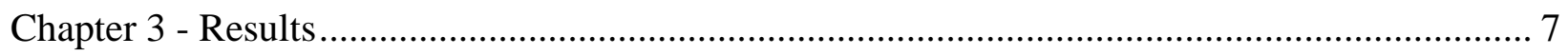

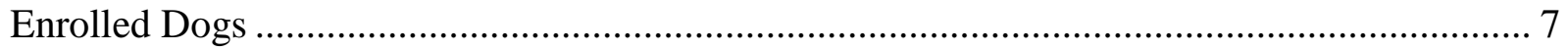

Plasma Cefovecin Concentration......................................................................................... 7

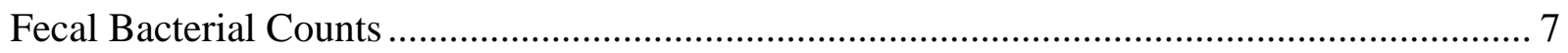

Antimicrobial Resistance ............................................................................................. 8



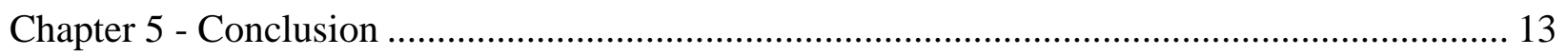

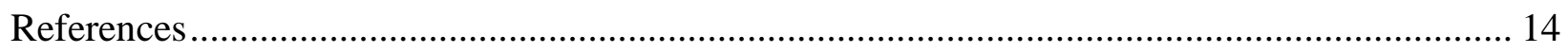

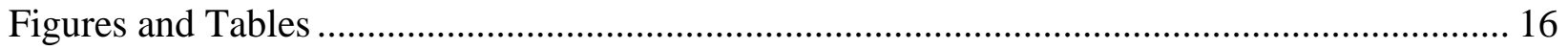




\section{List of Figures}

Figure 1. Absolute number of fecal E. coli presented as log CFU/g feces from treated and

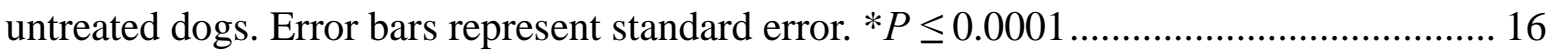

Figure 2. Absolute number of fecal cefovecin-resistant $E$. coli isolated from EC-CEF plates presented as $\log \mathrm{CFU} / \mathrm{g}$ feces from treated and untreated dogs. Error bars represent standard

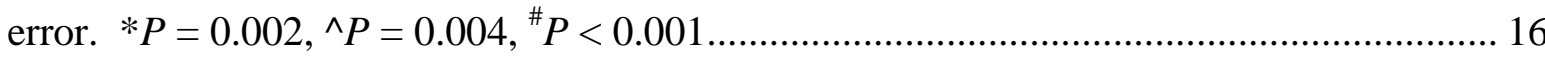

Figure 3. Percent of each enterococcal species by PCR on each sampling day from both groups combined.

Figure 4. Percent of fecal E. coli that were resistant to tested antimicrobials via the disc diffusion method on day 0 . No differences were documented between treated and untreated dogs. .. 18

Figure 5. Percent of fecal E. coli that were resistant to tested antimicrobials via the disc diffusion method on day 3. *Signifies $P<0.05$.

Figure 6. Percent of fecal E. coli that were resistant to tested antimicrobials via the disc diffusion

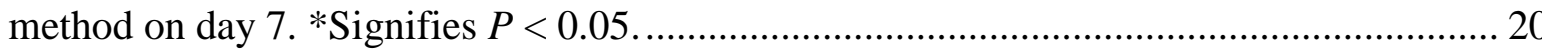

Figure 7. Percent of fecal E. coli that were resistant to tested antimicrobials via the disc diffusion method on day 14 . *Signifies $P<0.05$. 21

Figure 8. Percent of fecal E. coli that were resistant to tested antimicrobials via the disc diffusion method on day 28. *Signifies $P<0.05$.

Figure 9. Percent of fecal E. faecalis and E. faecium resistant to each antimicrobial, based on disc diffusion testing. q/d, quinupristin-dalfopristin. There was no significant difference between the treatment groups for each antimicrobial. 


\section{List of Tables}

Table 1. Sample preparation for the determination of cefovecin concentration in plasma and plasma standards.

Table 2. Mobile phase for the determination of cefovecin in plasma and plasma standards. A C18 column (Supelco Discovery, 50 x $2.1 \mathrm{~mm}, 5 \mu \mathrm{M}$, Sigma) achieved separation while maintained at $40{ }^{\circ} \mathrm{C}$

Table 3. Mass spectrometry settings for the determination of cefovecin concentration in plasma using cephalexin as the internal standard. The accuracy and coefficient of variation were determined on replicates of three at each of the following concentrations: $0.05,1$, and 50 $\mu \mathrm{g} / \mathrm{mL}$

Table 4. MIC range and $\mathrm{MIC}_{90}$ values for tested antimicrobials among cefovecin-susceptible and cefovecin-resistant E. coli collected on days 0,7 , and 28 . 


\section{Acknowledgements}

I would like to acknowledge my major professor Dr. Sanjeev Narayanan for always challenging me to do more; Dr. Katherine KuKanich for truly making this achievement possible; and, Dr. Gregory Grauer for all of his support during this project. Additional acknowledgement goes to the following in no particular order: Dr. Butch KuKanich, Dr. Hans Coetzee, and Mrs. Ellen Heinrich.

This project was supported by the Morris Animal Foundation (MAF). The content is solely the responsibility of the authors and does not necessarily represent the official views of the MAF. The MAF did not play any role in the design, data collection, analysis and interpretation, writing of the manuscript or decision to submit the manuscript for publication. This project was also supported in part by the Kansas State University Veterinary Research Scholar Program, and the College of Veterinary Medicine Departments of Anatomy and Physiology and Clinical Sciences. Valuable technical support was provided by Sailesh Menon, Deepti Pillai, Ludek Zurek, and Anu Ghosh.

This report was published by Elsevier and can be found using the following citation: Lawrence M., KuKanich K., KuKanich B., Heinrich E., Coetzee J.F., Grauer G., Narayanan S., 2013. Effect of cefovecin on the fecal flora of healthy dogs. The Veterinary Journal 198(1), 259266. 


\section{Chapter 1 - Introduction}

\section{Literature Review}

Cefovecin is a semi-synthetic third-generation long-acting cephalosporin. It is authorized for use in the European Union, New Zealand, and in several countries in South America and Asia. In the United States, it is US Food and Drug Administration approved and labeled for the treatment of superficial pyodermas, abscesses, and wounds in dogs. In dogs, cefovecin has a half-life of 5.5 days, allowing a single subcutaneous (SC) injection to provide up to 14 days of treatment (Stegemann et al., 2006a). Cefovecin undergoes minimal hepatic metabolism and is primarily excreted unchanged in the urine, but excretion of unchanged drug also occurs in the bile resulting in exposure to gastrointestinal (GI) flora (Stegemann et al., 2006a).

Cephalosporins have a significant effect on the fecal flora of cattle. Calves administered intramuscular (IM) ceftiofur hydrochloride had a significantly increased number of ceftriaxoneresistant fecal bacteria following treatment (Jiang et al., 2006). Cattle administered ceftiofur crystalline-free acid had a 2-week increase in multidrug-resistant (ceftiofur, ampicillin, chloramphenicol, tetracycline, and sulfisoxazole) Escherichia coli (Lowrance et al., 2007). Ceftiofur crystalline-free acid-treated cattle also had increased levels of fecal bla $a_{\mathrm{CMY}-2}$ carriage (Alali et al., 2009). The bla $a_{\mathrm{CMY}-2}$ gene encodes cephalosporin resistance via AmpC $\beta$-lactamase and has been identified among commensal and pathogenic bacteria within the GI flora of humans and animals (Forward et al., 2004; Alali et al., 2009). In vitro conjugation studies have documented plasmid transfer of the $b l a_{\mathrm{CMY}-2}$ gene within and between fecal genera, specifically E. coli and Salmonella spp. (Jiang et al., 2006). Bacteria containing the bla $a_{\mathrm{CMY}-2}$ gene produce extended spectrum beta lactamases and therefore carry resistance to all beta lactams licensed for use in dogs (Damborg et al., 2011). This can pose an animal health problem when selecting an antimicrobial to be effective in treating canine infections.

Few data exist regarding the effect of antimicrobials on canine fecal flora. Oral enrofloxacin administered to dogs suppressed fecal coliforms throughout treatment and slowly normalized within 8 days after termination of treatment (Trott et al., 2004). A separate study evaluating fecal E. coli from dogs found an association between multidrug resistance and history 
of receiving antimicrobials within 1 month of sampling, suggesting that selection pressure from antimicrobial therapy may influence the fecal flora (Stenske et al., 2009).

\section{Study Objectives}

The objectives of the present study were (1) to investigate the effect of cefovecin on the total number of coliforms and enterococci in the fecal flora of dogs, (2) to investigate the effect of cefovecin on the antimicrobial resistance of these bacteria, as determined by disc diffusion, minimum inhibitory concentration (MIC) determination, and presence of the antimicrobial resistance gene $b l a_{\mathrm{CMY}-2}$ on select isolates, and (3) to measure the plasma concentration of cefovecin on day 14 to ensure that untreated dogs did not inadvertently receive cefovecin. 


\section{Chapter 2 - Materials and Methods}

\section{Study Design and Enrolled Dogs}

The Kansas State University (KSU) Institutional Animal Care and Use Committee approved the study (protocol number 2941).

Fourteen young adult Beagles living in a closed research facility were enrolled. A sample size of at least six dogs in each group was calculated based on an estimated 3-log difference in bacterial count between treatment groups, with a $P$ value of 0.05 , and power of 80 (Trott et al., 2004). No dogs had received antimicrobials at any time prior to the study, and all dogs were deemed healthy based on a physical examination, complete blood count, biochemical profile, and fecal flotation examination.

Dogs were randomly divided, with blocking by age, using a random numbers table into a treated group and untreated group. Seven treated dogs received $8 \mathrm{mg} / \mathrm{kg}$ cefovecin (Convenia, Zoetis) SC on day 1. Seven untreated dogs received no therapy. Dogs were housed in separate runs with no direct contact in the month prior to and throughout the study. They ate a dry maintenance dog food and received no medication other than the cefovecin (treated dogs) during the study. Authors performing bacterial isolation, identification, enumeration, and susceptibility testing were blinded to treatment group designation.

\section{Bacterial Isolation}

One gram of fresh feces was collected from each of the $14 \mathrm{dogs}$, via rectal palpation with a sterile glove, on days $0,3,7,14$, and 28 . Feces were diluted 10 -fold in $0.1 \%$ peptone water and spread-plated on EC containing 4-methylumbelliferyl-beta-D-glucuronide agar (EC), Hektoen

enteric agar (HEA), and mEnterococcus agar (mENT). Additionally, diluted fecal samples were spread-plated onto these three media each containing $1 \mu \mathrm{g} / \mathrm{mL}$ cefovecin (EC-CEF, HEA-CEF, and mENT-CEF). EC plates were incubated at $44{ }^{\circ} \mathrm{C}$ for 24 hours, HEA plates at $37{ }^{\circ} \mathrm{C}$ for 24 hours, and mENT plates at $37^{\circ} \mathrm{C}$ for 48 hours.

\section{Bacterial Identification}

Isolates on EC plates were considered E. coli if they had the desired morphology, fluoresced under $366 \mathrm{~nm}$ ultraviolet light, were indole positive, and oxidase negative. 
Salmonella enrichment was performed by adding $1 \mathrm{~mL}$ of the $10^{-1}$ dilution to $9 \mathrm{~mL}$ Rappaport broth, incubating for 24 hours at $44^{\circ} \mathrm{C}$, plating on HEA and incubating for an additional 24 hours. Isolates on HEA were considered Salmonella if they were blue-green colonies that were indole negative and oxidase negative. Esculin hydrolysis was used to confirm isolates from mENT on the genus level as enterococci. A multiplex PCR assay was performed on three random enterococcal isolates grown on mENT plates per fecal sample, to determine the species as Enterococcus faecium or Enterococcus faecalis, as previously described (Kariyama et al., 2000; Poyart et al., 2000). Isolates of E. coli, Salmonella, and enterococci were enumerated as colony forming units (CFU) per gram of feces from each fecal sample. Isolates were stored in brain heart infusion broth with glycerol at $-80{ }^{\circ} \mathrm{C}$.

\section{Plasma Cefovecin Concentration Analysis}

Plasma samples were obtained on day 14 from each dog for the determination of cefovecin concentration using liquid chromatography (LC) (Shimadzu Prominence, Shimadzu

Scientific Instruments) with mass spectrometry (MS) (API 2000, Applied Biosystems). Plasma standards and samples were processed according to Table 1; the LC settings are described in Table 2, and the MS settings in Table 3.

\section{Antimicrobial Susceptibility Testing}

To determine the absolute number of cefovecin-resistant fecal bacteria after administration of cefovecin, E. coli, Salmonella, and enterococci were enumerated from ECCEF, HEA-CEF, and mENT-CEF plates, respectively, at each sampling time. Antimicrobial susceptibility testing using the disc diffusion method with Mueller-Hinton agar was performed on up to five randomly selected $E$. coli isolates from each media not containing cefovecin per fecal sample on each sampling day. E. coli were tested for susceptibility to 12 antimicrobials, enterococci were tested for susceptibility to 10 antimicrobials, and Salmonella were not tested for susceptibility.

Determination of the MIC was performed on five E. coli isolates from each EC plate on days 0,7 , and 28 for cefovecin, ceftriaxone, enrofloxacin, gentamicin, and imipenem. Testing was performed and interpreted in accordance with the recommendations of the Clinical Laboratory Standards Institute (CLSI), using zone diameters and interpretive breakpoints for Enterobacteriaceae and Enterococcus spp. for dogs when available, otherwise guidelines for 
humans were used (CLSI M31-A3 2008; CLSI M100-S20 2010). The CLSI has not approved zone diameters or MIC breakpoint criteria for cefovecin, thus breakpoints used were based on recommendations from Zoetis (disc diffusion: $\mathrm{S} \geq 24, \mathrm{I} 21-23, \mathrm{R} \leq 20 \mathrm{~mm}$; $\mathrm{MIC} \mathrm{S} \leq 2, \mathrm{I} 4, \mathrm{R} \geq 8$ $\mu \mathrm{g} / \mathrm{mL})$.

Three randomly selected $E$. coli isolates from EC plates and three randomly selected $E$. coli isolates from EC-CEF plates from each dog on each day were tested using PCR for the presence of the $b l a_{\mathrm{CMY}-2}$ gene (not necessarily the same isolates as those selected for susceptibility testing). E. coli DNA was isolated by boil-prep, and spectrophotometry was used to ensure nucleic acid concentration and quality from each E. coli isolate. Real-time PCR was performed to determine the presence of the $b l a_{\mathrm{CMY}-2}$ gene compared to the $e u b$ housekeeping gene in the overall fecal population of each dog on each sampling day. Total DNA from $200 \mu \mathrm{g}$ of feces was extracted using QIAamp DNA stool mini kit (Qiagen), following the manufacturer's protocol.

The $b l a_{\mathrm{CMY}-2}$ gene was amplified using forward primer $5^{1} \mathrm{GACAGCCTCTTTCTCCACA}$ (reference number 59972060), and reverse primer $5^{1}$ GAATAGCCTGCTCCTGCATC (reference number 59972061) (Zhao et al, 2001). PCR amplification (denaturation for 30 seconds at $95{ }^{\circ} \mathrm{C}$, primer annealing for 30 seconds at $55^{\circ} \mathrm{C}$, and extension for 30 seconds at $72{ }^{\circ} \mathrm{C}$ ) consistently yields a product size of $101 \mathrm{bp}$ and has been successfully used in DNA from pure cultures or total fecal DNA (Zhao et al, 2001).

\section{Statistical Method}

Mean age and body weight of enrolled dogs with one standard deviation were calculated, and a Student's $t$ test was used to compared these values between treated and untreated groups. A random effects-mixed model was used to analyze bacterial counts in a repeated measures format, and data were presented as mean CFU/gram feces \pm standard error of the mean. Distribution of enterococcal species, antimicrobial resistance testing by disc diffusion, and presence of the $b l a_{\mathrm{CMY}-2}$ gene in $E$. coli isolates were analyzed using the Fisher's exact test and relative risk. $\mathrm{MIC}$ results were reported as range and $\mathrm{MIC}_{90}$. The presence of the $b l a_{\mathrm{CMY}-2}$ gene from the total fecal population was achieved by comparing the ratio of the $b l a_{\mathrm{CMY}-2}$ gene to the universal eubacterial $e u b$ gene (bla:eub) in collected fecal samples on each day using a random effectsmixed model in a repeated measures format. A $P$-value of $<0.05$ was considered significant for 
all analyses. Statistical analyses were evaluated using the commercial software program JMP 9 (SAS Institute). 


\section{Chapter 3 - Results}

\section{Enrolled Dogs}

Six male intact and eight female intact Beagles were enrolled. Mean age was 11.57 \pm 0.94 months and body weight was $9.69 \pm 1.26 \mathrm{~kg}$; there was no difference in age $(P=0.589)$ or body weight $(P=0.921)$ between groups. No adverse clinical signs were noted in any dog.

\section{Plasma Cefovecin Concentration}

The average plasma cefovecin concentration on day 14 in treated dogs was $9.59 \mu \mathrm{g} / \mathrm{mL}$ (range 3.20-12.90 $\mu \mathrm{g} / \mathrm{mL}$ ). Cefovecin was not detected in any untreated dogs.

\section{Fecal Bacterial Counts}

On day 0 , a mean of $6.7 \pm 1.8 \times 10^{6} \mathrm{CFU}$ of $E$. coli per gram of feces was isolated, with no difference identified between treated and untreated dogs (Figure 1). Thereafter, a significant time * treatment group interaction was identified $(P<0.0001)$, and on day 3 the absolute number of fecal E. coli dropped significantly in the treated group (Figure 1). Based on the absolute number of $E$. coli grown on media containing cefovecin, resistance to cefovecin among $E$. coli increased throughout the study and was significantly greater in the treated vs. untreated dogs on days 7, 14, and 28 (Figure 2).

Salmonella isolation was rare, even with enrichment. Salmonella spp. were isolated from the feces of six dogs ( 3 treated, 3 untreated), but no effect of treatment $(P=1.000)$ or interaction between treatment and time $(P=0.676)$ was identified. Cefovecin-resistant Salmonella were isolated from one treated and one untreated dog on day 28 but not on other days.

On day 0 , a mean of $1.3 \pm 0.7 \times 10^{7} \mathrm{CFU}$ of enterococci per gram of feces was found, with no difference between treated and untreated $\operatorname{dogs}(P=1.000)$. However, a significant time * treatment group interaction was identified $(P=0.002)$, with more enterococci isolated from feces of treated dogs compared with untreated dogs on day $7(P=0.023)$. No differences were seen in enumeration of cefovecin-resistant enterococci between groups at any time point. All enterococcal isolates were identified with PCR as either E. faecalis or E. faecium. E. faecalis were more common than E. faecium in both groups on day 0, but the percentages of E. faecalis isolates decreased and E. faecium isolates increased until day 14 (Figure 3). By day 3 isolates 
from treated dogs $(12 / 21,66.7 \%)$ were 14 times as likely as from untreated dogs $(1 / 21,4.8 \%)$ to be E. faecium ( $P<0.0001,95 \%$ CI 2.0-97.5), but by day 7 there was no difference in species distribution between groups $(P=0.520)$, with feces from both treated and untreated dogs having E. faecium percentage peaking at 14 days.

\section{Antimicrobial Resistance}

Based on disc diffusion testing, there were no differences in susceptibility between $E$. coli (EC plates) from treated and untreated dogs on day 0, but resistance to cefovecin was high in both groups (Figure 4). On days 3, 7, 14, and 28, significant differences were observed, with fecal $E$. coli from treated dogs being more likely to be resistant to numerous tested antimicrobials than feces from untreated dogs (Figures 5-8).

Based on disc diffusion testing, from the population of E. coli that grew on media containing cefovecin (EC-CEF), all isolates from all treated and untreated dogs on all sampling days were resistant to cefovecin, as well as to ampicillin, cefazolin, and cefpodoxime. Of tested isolates from treated and untreated dogs, $>97 \%$ were resistant to amoxicillin-clavulanic acid at each sampling time, and $>33 \%$ were resistant to ceftiofur. In these isolates, ceftriaxone resistance increased in both groups from day 0 (20\% treated, $28 \%$ untreated) to day $28(82.9 \%$ treated, $77.1 \%$ untreated), with no group effect. All E. coli isolates from EC-CEF were susceptible (via disc diffusion) to gentamicin and imipenem, while resistance to doxycycline, enrofloxacin, and TMS increased throughout the study, with no group effect.

MIC was performed on E. coli isolated from EC plates from treated and untreated dogs on days 0,7 , and 28 for cefovecin, ceftriaxone, enrofloxacin, gentamicin, and imipenem, and compared based on previous categorization (via disc diffusion) of E. coli as being cefovecinsusceptible or cefovecin-resistant (Table 4).

At baseline, presence of the $b l a_{\mathrm{CMY}-2}$ gene was isolated from the fecal E. coli of $14.3 \%$ $(3 / 21)$ of treated and $4.8 \%(1 / 21)$ of untreated dogs $(P=0.606)$. No treatment group differences were noted until day 28 when fecal E. coli (from EC plates) from treated dogs $(62 \%, 13 / 21)$ were 3.25 times more likely $(95 \%$ CI 1.27-8.35) than fecal E. coli from untreated dogs $(19 \%, 4 / 21)$ to carry the bla $a_{\mathrm{CMY}-2}$ gene $(P=0.010)$. While the percent of fecal $E$. coli containing the bla $a_{\mathrm{CMY}-2}$ gene increased over the study period (14-76\%) among those isolates tested from EC-CEF plates, no significant treatment group differences were documented. The ratio of the $b l a_{\mathrm{CMY}-2}$ gene to 
the universal eubacterial gene, bla:eub, was then assessed in the total fecal bacterial population of enrolled dogs. Contrary to what was found when assessing presence of bla $a_{\mathrm{CMY}-2}$ in E. coli alone, the feces of untreated dogs had a higher mean bla:eub (2.714 \pm 0.84$)$ compared with the feces of treated dogs $(1.667 \pm 0.28)$ on day $28(P<0.0001)$; no differences were found on other days, and there was no significant change over time $(P=0.145)$.

Treatment group had no effect on antibiotic susceptibility within E. faecalis or E. faecium isolates over the study time period, with the exception of day 28 E. faecium isolates from treated dogs $(94.4 \%)$ which were more frequently resistant to tigecycline than isolates from untreated dogs $(54.6 \%)(P=0.019)$. Percent resistance to each antimicrobial of all E. faecalis and $E$. faecium isolates together are presented in Figure 9. 


\section{Chapter 4 - Discussion}

Plasma cefovecin concentrations confirmed that no untreated dog inadvertently received cefovecin. Cefovecin concentrations in treated dogs on day $14(3.20-12.90 \mu \mathrm{g} / \mathrm{mL})$ were similar to a previous study which reported $5.6 \pm 1.8 \mu \mathrm{g} / \mathrm{mL}$ and $15.2 \pm 3.2 \mu \mathrm{g} / \mathrm{mL}$ in two groups of dogs 14 days after administration (Stegemann et al., 2006a). A second study reported the in vitro $\mathrm{MIC}_{90}$ of cefovecin for $E$. coli isolates from the United States was $1 \mu \mathrm{g} / \mathrm{mL}$; however the correlation between in vitro $\mathrm{MIC}_{90}$ and in vivo plasma concentration, especially for highly protein bound drugs such as cefovecin, has not been determined (Stegemann et al., 2006b).

Due to the bacterial isolation and identification techniques used, only typical Salmonella, E. coli, and enterococci were selected for analysis. Any atypical isolates that might have been present were not analyzed.

The significant decline in total number of E. coli within 72 hours of cefovecin administration was similar to that seen with oral enrofloxacin administration to dogs (Trott et al., 2004). However, in that study, total coliform numbers remained below detectable limits throughout antimicrobial administration (21 days) followed by a return to untreated levels within 8 days after ceasing administration, while in the current study total $E$. coli levels returned to levels consistent with untreated dogs by day 7 despite treated dogs having measurable cefovecin in their plasma on day 14 (Trott et al., 2004). One potential explanation would be differences in gastrointestinal exposure of cefovecin and enrofloxacin.

The absolute number of cefovecin-resistant $E$. coli increased over the study period in untreated dogs as well as treated dogs. The authors hypothesized that this was caused by fecal oral cross-contamination which may have occurred between the two groups of dogs despite deliberate efforts to prevent this, including prohibiting direct contact among dogs, housing in runs with solid walls to prevent fecal spread between kennels, and keeping kennels clean. Suspected cross-contamination is a limitation of the present study, and more aggressive attempts to prevent any contact between the dogs or their feces should be made for future research studies (i.e. separate wards, foot baths, etc.). However, the effects of suspected cross-contamination may be relevant to a clinical hospital setting, where dogs are in kennels next to each other and similar precautions (no direct contact, keeping kennels clean, wearing gloves) are taken to minimize 
disease transmission. Thus, it is reasonable to believe that resistant bacteria could be shed from a patient receiving antimicrobials and transferred to a dog in a nearby kennel, which could be deleterious if the recipient is highly susceptible to infection, such as an immunosuppressed patient. A public health risk could also be inferred, as resistant fecal bacteria could be spread to the veterinary staff or dog's owner.

Disc diffusion and MIC testing of $E$. coli from EC plates identified a high percentage of cefovecin-resistant E. coli at baseline and throughout the study in treated and untreated dogs. The most reliable measure of cefovecin resistance in this study was from enumeration of absolute numbers of cefovecin-resistant $E$. coli grown on EC-CEF media at each sampling day, and this technique found a lower rate of cefovecin-resistance at baseline in both groups. One explanation for this discrepancy could be that without CLSI approved zone diameters and breakpoint criteria, the manufacturer-recommended breakpoint criteria may need to be re-evaluated. It was also possible that the enrolled dogs may have had cefovecin resistance prior to the study from maternal or environmental exposure to resistant bacteria.

Significant differences occurred between treated and untreated dogs in the percentage of resistant $E$. coli isolates via disc diffusion to other beta-lactams after administration of cefovecin. The difference in $b l a_{\mathrm{CMY}-2}$ carriage identified on day 28 from fecal E. coli of treated and untreated dogs may indicate that cefovecin treatment selects for E. coli producing a plasmidborne CMY-2 beta-lactamase. A study of dogs with pyoderma also found that treatment with cephalexin resulted in fecal E. coli from 8/13 dogs carrying plasmid-mediated bla $a_{\mathrm{CMY}-2}$ (Damborg et al., 2011). Acquisition of this gene may explain the cross resistance seen among $\beta$ lactams in this study. Furthermore, co-residence of multiple resistance genes has been documented in $E$. coli carrying the $b l a_{\mathrm{CMY}-2}$ gene, which may help to explain the resistance seen in the fecal E. coli of the present study to doxycycline, enrofloxacin, gentamicin, and imipenem (Winokur et al., 2001).

The significant increase in the number of enterococci isolated from treated dogs compared with untreated dogs on day 7 was not surprising, as enterococci are not susceptible to cefovecin and would have a competitive advantage when other enteric flora (E. coli) are eliminated by cefovecin. At day 0 of this study, E. faecalis was the predominant enterococcal species $(88 \%)$, which is consistent with a previous report of canine rectal swabs $(n=86)$ that had a species distribution of E. faecalis (60\%), E. hirae (15\%), and E. faecium (8\%) (Jackson et al., 
2009). It is possible that cefovecin contributed to the change in species distribution towards a higher percentage of E. faecium, but no mechanism for this effect is known; sampling bias from random selection of colonies may have also played a role in this change. 


\section{Chapter 5 - Conclusion}

This study documented that cefovecin administration impacted both the number and antimicrobial resistance of fecal E. coli in healthy dogs. However, the clinical implications of the results are unknown as the work was performed using young healthy dogs in a research setting. Future studies are recommended to assess the effect of other cephalosporins, route of administration, and other classes of antimicrobials on the canine gastrointestinal flora, as well as the effect of these drugs on the flora of clinically ill patients. The results from this study suggest that antimicrobials should only be prescribed to dogs when benefits to the patient clearly outweigh the risks. 


\section{References}

Alali WQ, Scott HM, Norby B, Gebreyes W, Loneragan GH. 2009. Quantification of the BlaCMY-2 in feces from beef feedlot cattle administered three different doses of ceftiofur in a longitudinal controlled feed trial. Foodborne Pathogens and Disease 6, 917924.

Clinical and Laboratory Standards Institute. 2008. Performance Standards for Antimicrobial Disk and Dilution Susceptibility Tests for Bacteria Isolated from Animals. Approved Standard CLSI Document M31-A3.vol. 28, Third Ed. Clinical and Laboratory Standards Institute, Wayne, Pa, pp.1-99.

Clinical and Laboratory Standards Institute. 2010. Performance Standards for Antimicrobial Susceptibility Testing; Twentieth Informational Supplement. CLSI Document M100-S20, vol. 30. Clinical and Laboratory Standards Institute, Wayne, Pa, pp.76-79.

Damborg, P., Gaustad, I.G., Olsen, J.E., Guardabassi, L., 2011. Selection of CMY-2 producing Escherichia coli in the faecal flora of dogs treated with cephalexin. Veterinary Microbiology 151, 404-408.

Forward, K.R., Matheson, K.M., Hiltz, M., Musgrave, H., Poppe, C., 2004. Recovery of cephalosporin-resistant Escherichia coli and Salmonella from pork, beef, and chicken marketed in Nova Scotia. The Canadian Journal of Infectious Disease and Medical Microbiology 15, 226-230.

Jiang, X., Yang, H., Dettman, B., Doyle, M.P., 2006. Analysis of fecal microbial flora for antibiotic resistance in ceftiofur-treated calves. Foodborne Pathogens and Disease 3, 355365.

Jackson, C.R., Fedorka-Cray, P.J., Davis, J.A., Barrett, J.B., Frye, J.G., 2009. Prevalence, species distribution, and antimicrobial resistance of enterococci isolated from dogs and cats in the United States. Journal of Applied Microbiology 107, 1269-1278.

Kariyama, R., Mitsuhata, R., Chow, J.W., Clewell, D.B., Kumon, H., 2000. Simple and reliable multiplex PCR assay for surveillance isolates of vancomycin-resistant enterococci. Journal of Clinical Microbiology 38, 3092-3095.

Lowrance, T.C., Loneragan, G.H., Kunze, D.J., Platt, T.M., Ives, S.E., Scott, H.M., Norby, B., Echeverry, A., Brashears, M.M., 2007. Changes in antimicrobial susceptibility in a 
population of Escherichia coli isolated from feedlot cattle administered ceftiofur crystalline-free acid. American Journal of Veterinary Research 68, 501-507.

Poyart, C., Quesnes, G., Trieu-Cuot, P., 2000. Sequencing the gene encoding manganesedependent superoxide dismutase for rapid species identification of enterococci. Journal of Clinical Microbiology 38, 415-418.

Stegemann, M.R., Sherington, J., Blanchflower, S., 2006a. Pharmacokinetics and pharmacodynamics of cefovecin in dogs. Journal of Veterinary Pharmacology and Therapeutics 29, 501-511.

Stegemann, M.R., Passmore, C.A., Sherington, J., Lindeman, C.J., Papp, G., Weigel, D.J., Skogerboe, T.L., 2006b. Antimicrobial activity and spectrum of cefovecin, a new extended-spectrum cephalosporin, against pathogens collected from dogs and cats in Europe and North America. Antimicrobial Agents and Chemotherapy 50, 2006b. 50, 2286-2292.

Stenske, K.A., Bemis, D.A., Gillespie, B.E., D’Souza, D.H., Oliver, S.P., Draughon, F.A., Matteson, K.J., Bartges, J.W., 2009. Comparison of clonal relatedness and antimicrobial susceptibility of fecal Escherichia coli from healthy dogs and their owners. American Journal of Veterinary Research 70, 1108-1116.

Trott, D.J., Filippich, L.J., Bensink, J.C., Downs, M.T., McKenzie, S.E., Townsend, K.M., Moss, S.M., Chinn, J.J., 2004. Canine model for investigating the impact of oral enrofloxacin on commensal coliforms and colonization with multidrug-resistant Escherichia coli. Journal of Medical Microbiology 53, 1-5.

Winokur, P.L., Vonstein, D.L., Hoffman, L.J., Uhlenhopp, E.K., Doern, G.V., 2001. Evidence for transfer of CMY-2 AmpC $\beta$-lactamase plasmids between Escherichia coli and Salmonella isolates from food animals and humans. Antimicrobial Agents Chemotherapy 45, 2716-2722.

Zhao, S., White, D.G., McDermott, P.F., Friedman, S., English, L., Ayers, S., Meng, J., Maurer, J.J., Holland, R., Walker, R.D., 2001. Identification and expression of cephamycinase bla(CMY) genes in Escherichia coli and Salmonella isolates from food animals and ground meat. Antimicrobial Agents and Chemotherapy 45, 3647-3650. 


\section{Figures and Tables}

Figure 1. Absolute number of fecal $E$. coli presented as $\log \mathrm{CFU} / \mathrm{g}$ feces from treated and untreated dogs. Error bars represent standard error. $* \boldsymbol{P} \leq \mathbf{0 . 0 0 0 1}$

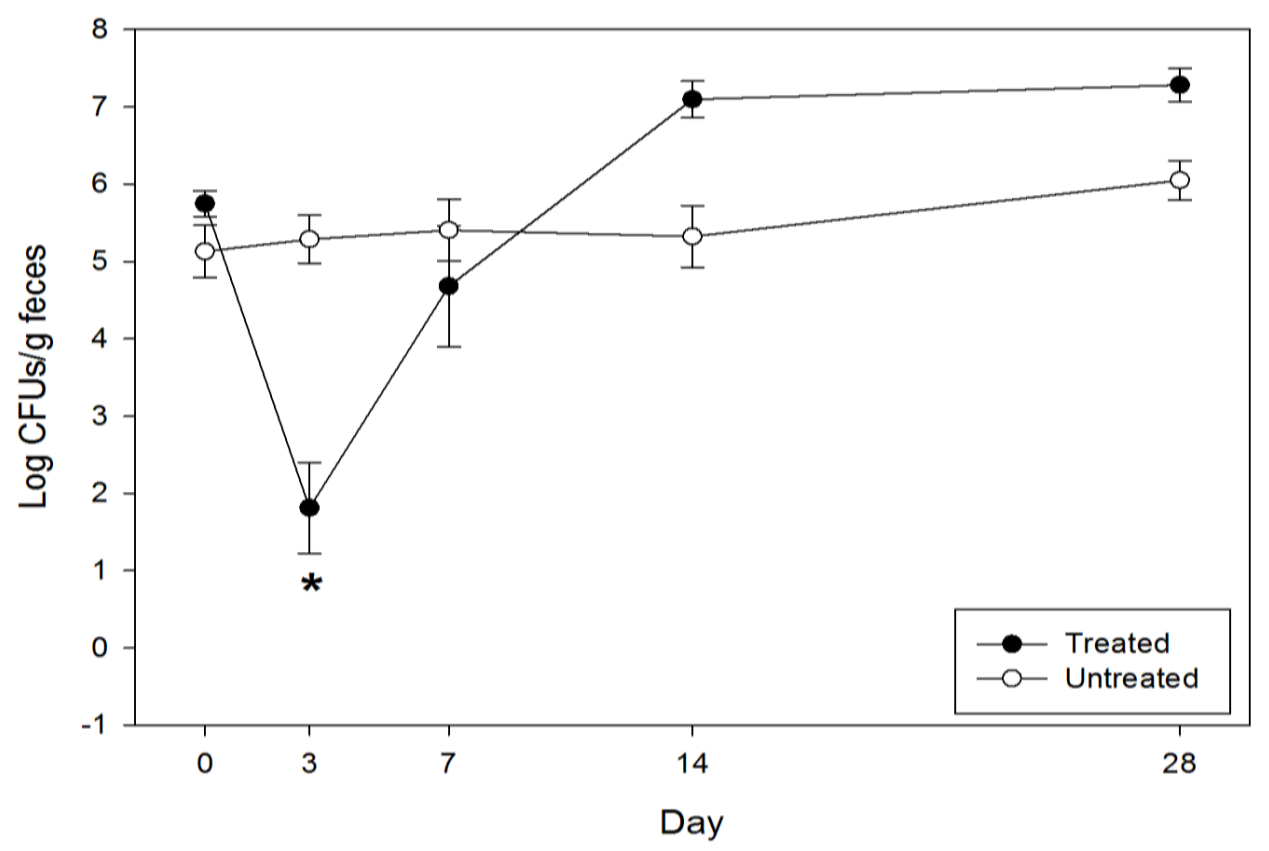

Figure 2. Absolute number of fecal cefovecin-resistant $E$. coli isolated from EC-CEF plates presented as $\log \mathrm{CFU} / \mathrm{g}$ feces from treated and untreated dogs. Error bars represent standard error. $* P=0.002, \wedge P=0.004,{ }^{\#} P<0.001$.

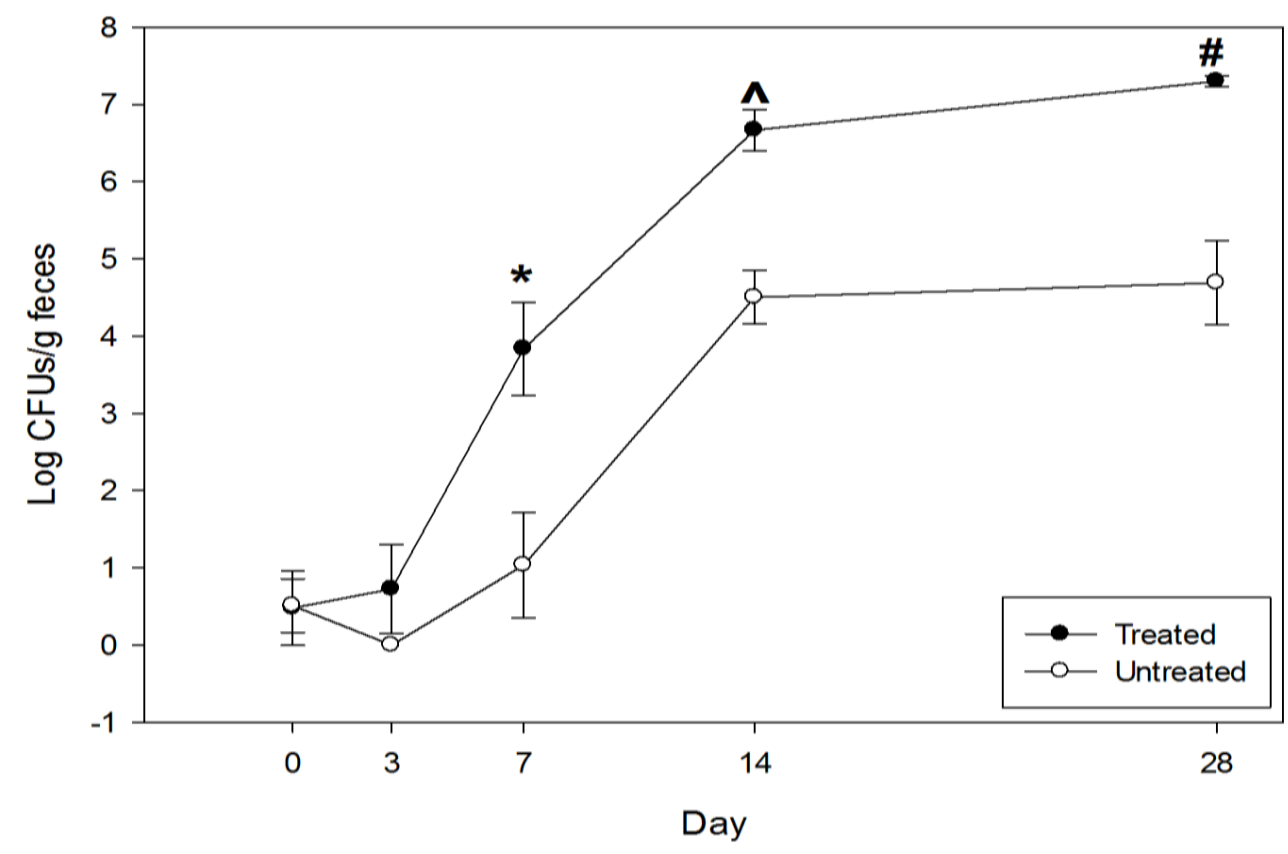


Figure 3. Percent of each enterococcal species by PCR on each sampling day from both groups combined.

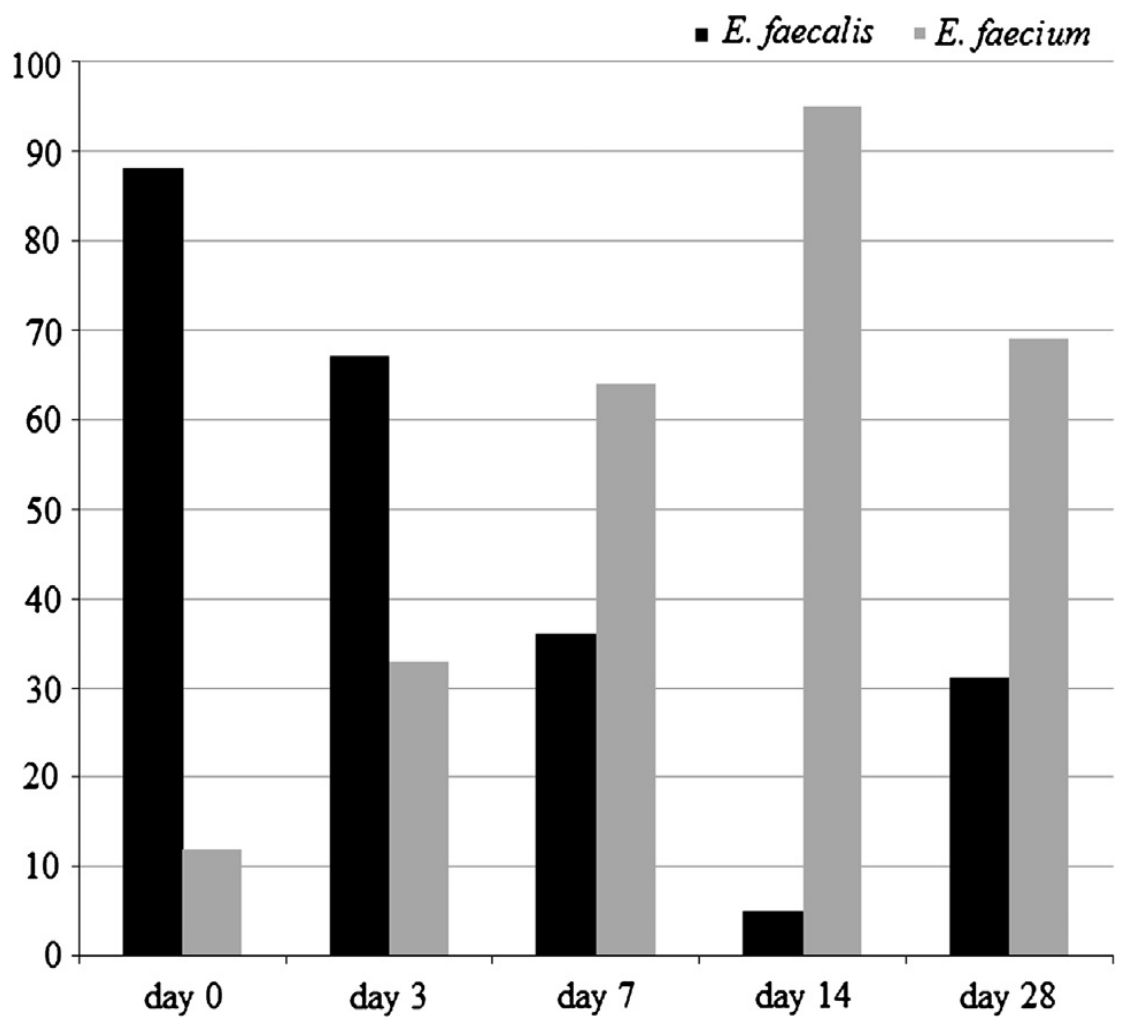


Figure 4. Percent of fecal $E$. coli that were resistant to tested antimicrobials via the disc diffusion method on day 0 . No differences were documented between treated and untreated dogs.

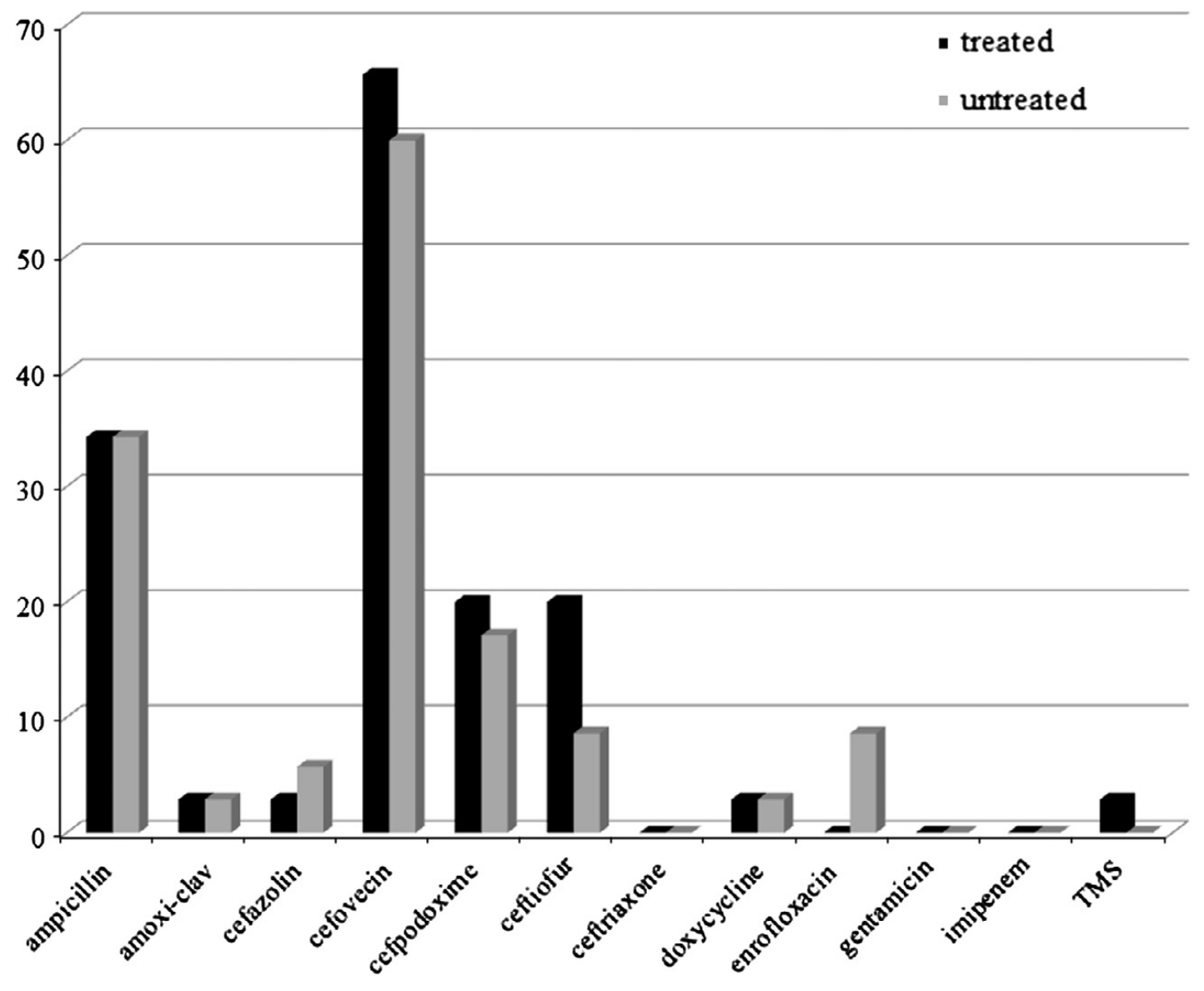


Figure 5. Percent of fecal $E$. coli that were resistant to tested antimicrobials via the disc diffusion method on day 3 . *Signifies $P<0.05$.

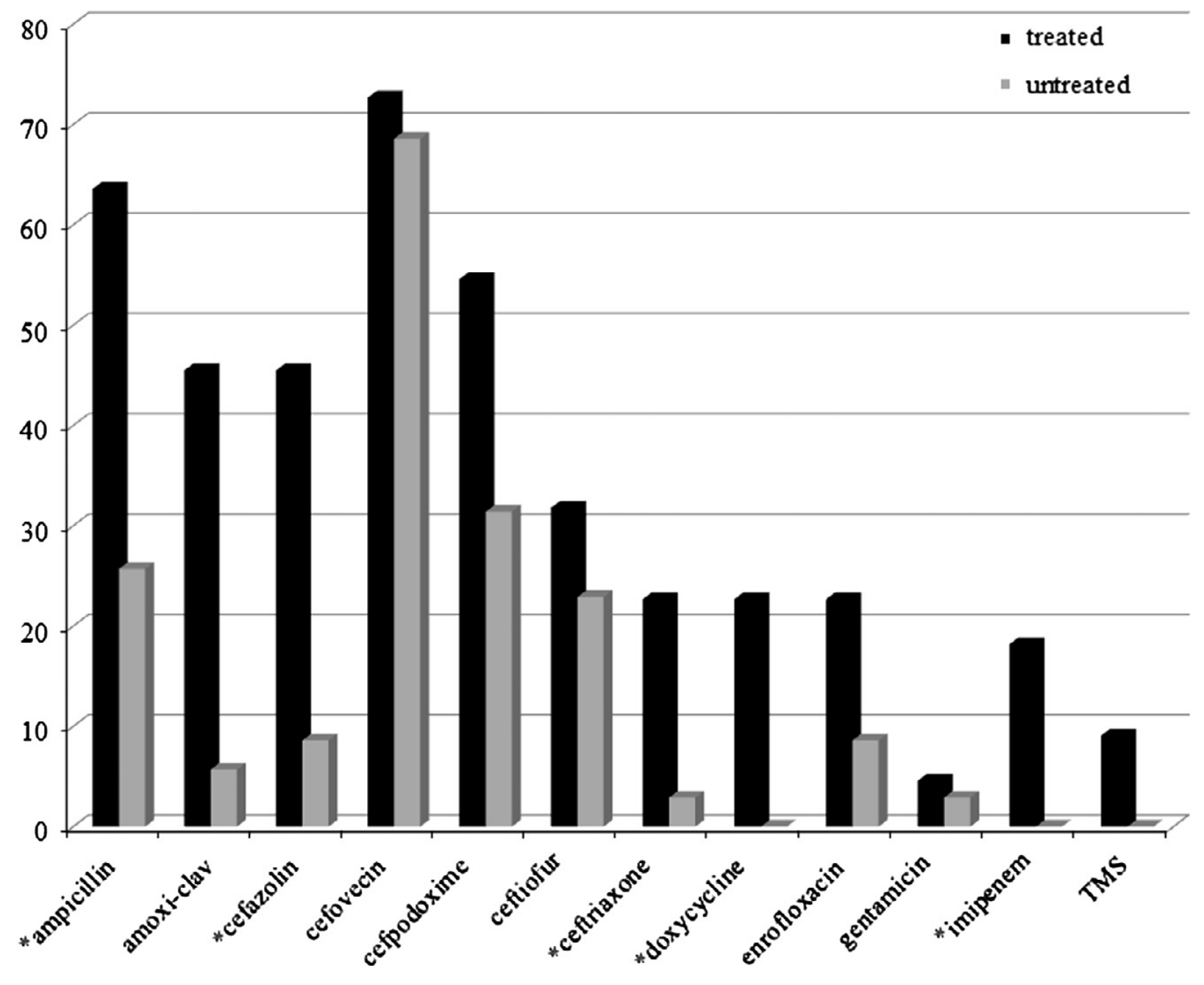


Figure 6. Percent of fecal $E$. coli that were resistant to tested antimicrobials via the disc diffusion method on day 7. *Signifies $P<0.05$.

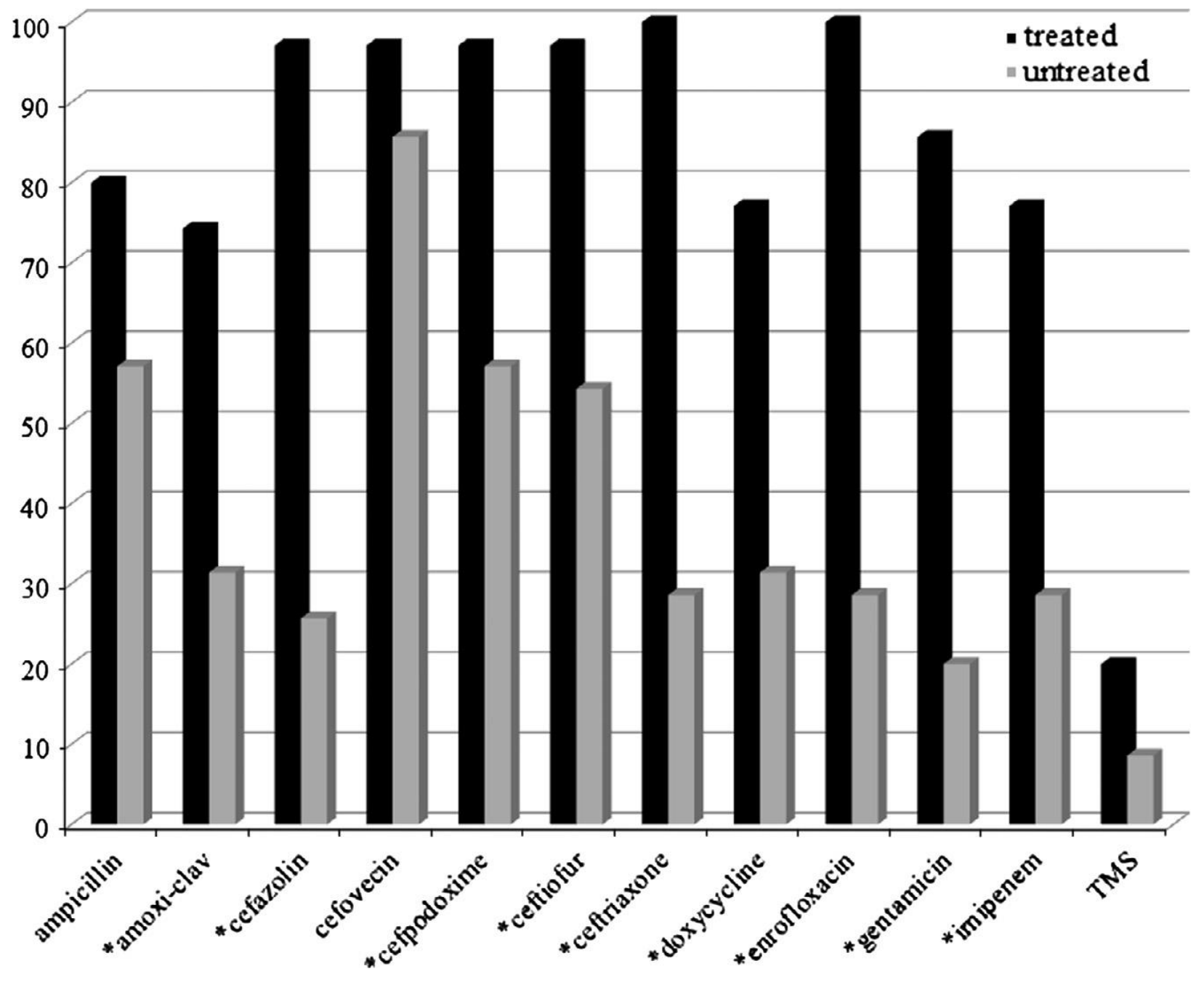


Figure 7. Percent of fecal $E$. coli that were resistant to tested antimicrobials via the disc diffusion method on day 14 . *Signifies $P<0.05$.




Figure 8. Percent of fecal $E$. coli that were resistant to tested antimicrobials via the disc diffusion method on day 28 . *Signifies $P<0.05$.

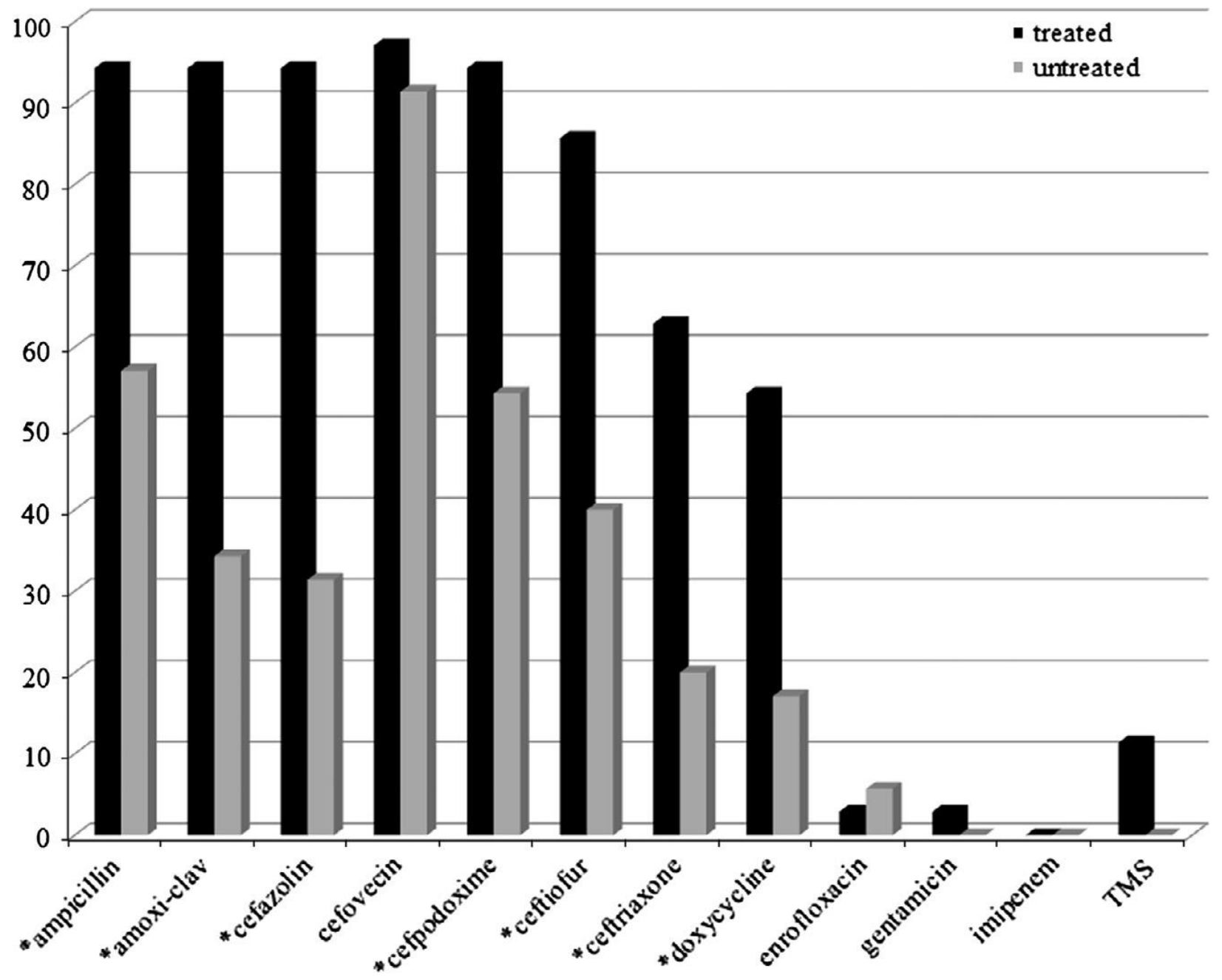


Figure 9. Percent of fecal $E$. faecalis and E. faecium resistant to each antimicrobial, based on disc diffusion testing. q/d, quinupristin-dalfopristin. There was no significant difference between the treatment groups for each antimicrobial.

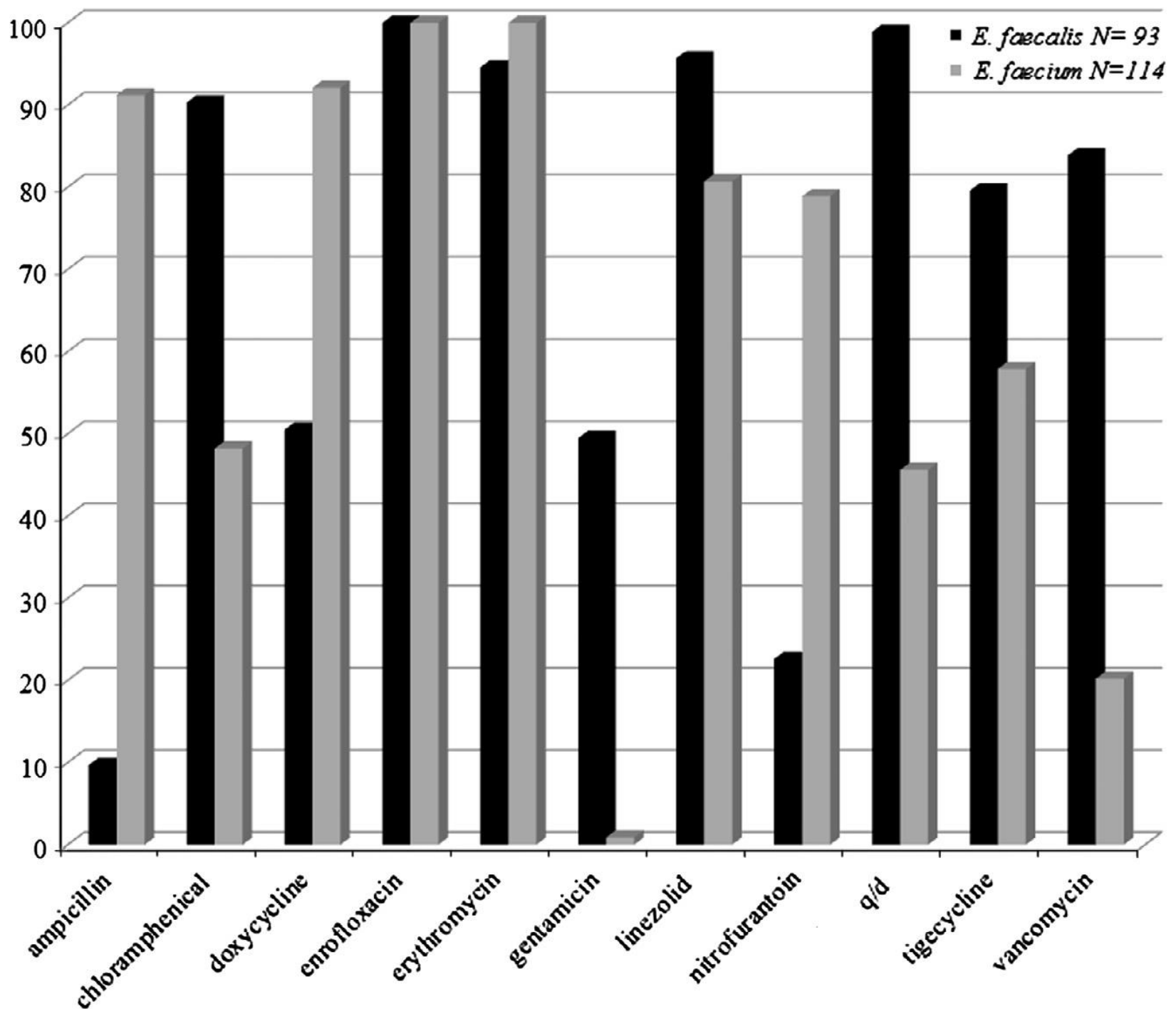


Table 1. Sample preparation for the determination of cefovecin concentration in plasma and plasma standards.

\begin{tabular}{|l|l|}
\hline Step & \\
\hline 1 & Add $0.1 \mathrm{~mL}$ plasma or plasma standard to microcentrifuge tube \\
\hline 2 & $\begin{array}{l}\text { Add } 0.4 \mathrm{~mL} \text { methanol containing cephalexin } 0.5 \mu \mathrm{g} / \mathrm{mL} \text { to } \\
\text { microcentrifuge tube }\end{array}$ \\
\hline 3 & Vortex microcentrifuge tube for $5 \mathrm{~s}$ \\
\hline 4 & Centrifuge microcentrifuge tube at $15,000 \mathrm{~g}$ for $5 \mathrm{~min}$ \\
\hline 5 & Transfer $0.2 \mathrm{~mL}$ supernatant to injection vial \\
\hline
\end{tabular}

Table 2. Mobile phase for the determination of cefovecin in plasma and plasma standards. A C18 column (Supelco Discovery, 50 x $2.1 \mathrm{~mm}, 5 \mu \mathrm{M}$, Sigma) achieved separation while maintained at $40{ }^{\circ} \mathrm{C}$.

\begin{tabular}{|l|l|l|}
\hline & \multicolumn{2}{|l|}{ Mobile Phase } \\
\hline Time $(\mathrm{min})$ & $\mathrm{A}=$ Acetonitrile & $\mathrm{B}=0.1 \%$ formic acid in water \\
\hline 0 & $5 \%$ & $95 \%$ \\
\hline 1 & $20 \%$ & $80 \%$ \\
\hline 3.5 & $5 \%$ & $95 \%$ \\
\hline 5.5 & $5 \%$ & $95 \%$ \\
\hline
\end{tabular}

Table 3. Mass spectrometry settings for the determination of cefovecin concentration in plasma using cephalexin as the internal standard. The accuracy and coefficient of variation were determined on replicates of three at each of the following concentrations: $0.05,1$, and $50 \mu \mathrm{g} / \mathrm{mL}$.

\begin{tabular}{|l|l|l|l|l|l|}
\hline & $\begin{array}{l}\text { Qualifying ion } \\
(\mathrm{m} / \mathrm{z})\end{array}$ & $\begin{array}{l}\text { Quantifying ion } \\
(\mathrm{m} / \mathrm{z})\end{array}$ & Accuracy & $\begin{array}{l}\text { Coefficient of } \\
\text { variation }\end{array}$ & $\begin{array}{l}\text { Linear standard } \\
\text { curve range }\end{array}$ \\
\hline Cefovecin & 454.08 & 241.00 & $99.6 \%$ & $3.8 \%$ & $0.05-50 \mu \mathrm{g} / \mathrm{mL}$ \\
\hline Cephalexin & 348.09 & 158.00 & N/A & N/A & N/A \\
\hline
\end{tabular}

N/A not applicable

Table 4. MIC range and $\mathrm{MIC}_{90}$ values for tested antimicrobials among cefovecinsusceptible and cefovecin-resistant $E$. coli collected on days 0,7 , and 28.

\begin{tabular}{|l|l|l|l|l|l|l|}
\hline & Day 0 & Day 0 & Day 7 & Day 7 & Day 28 & Day 28 \\
& Cef-S & Cef-R & Cef-S & Cef-R & Cef-S & Cef-R \\
& $\mathrm{N}=26$ & $\mathrm{~N}=44$ & $\mathrm{~N}=6$ & $\mathrm{~N}=64$ & $\mathrm{~N}=4$ & $\mathrm{~N}=66$ \\
\hline Cefovecin & Range & Range & Range & Range & Range & Range \\
& $<0.625-$ & $<0.625-$ & $1.25-$ & $1.25->200$ & $60->200$ & $20->200$ \\
\hline
\end{tabular}




\begin{tabular}{|c|c|c|c|c|c|c|}
\hline & $\begin{array}{l}10 \\
\mathrm{MIC}_{90} 10\end{array}$ & $\begin{array}{l}200 \\
\text { MIC }_{90} 60\end{array}$ & $\begin{array}{l}>200 \\
\text { MIC } 90 \\
\text { N/A }\end{array}$ & $\begin{array}{l}\mathrm{MIC}_{90} \\
>200\end{array}$ & $\begin{array}{l}\text { MIC }_{90} \\
\text { N/A }\end{array}$ & $\begin{array}{l}\text { MIC }_{90} \\
>200\end{array}$ \\
\hline Ceftriaxone & $\begin{array}{l}\text { Range } \\
<0.625-5 \\
\text { MIC }_{90} 5\end{array}$ & $\begin{array}{l}\text { Range } \\
<0.625-5 \\
\text { MIC }_{90} 5\end{array}$ & $\begin{array}{l}\text { Range } \\
1.25-2.5 \\
\text { MIC }_{90} \\
\text { N/A }\end{array}$ & $\begin{array}{l}\text { Range } \\
0.25->200 \\
\mathrm{MIC}_{90} \\
>200\end{array}$ & $\begin{array}{l}\text { Range } \\
1.25-10 \\
\text { MIC }_{90} \\
\text { N/A }\end{array}$ & $\begin{array}{l}\text { Range } \\
1.25-20 \\
\text { MIC }_{90} 20\end{array}$ \\
\hline Enrofloxacin & $\begin{array}{l}\text { Range } \\
0.5-16 \\
\text { MIC }_{90} 2\end{array}$ & $\begin{array}{l}\text { Range } \\
0.5-16 \\
\text { MIC }_{90} 16\end{array}$ & $\begin{array}{l}\text { Range } \\
2-16 \\
\text { MIC }_{90} \\
\text { N/A }\end{array}$ & $\begin{array}{l}\text { Range } \\
0.5->200 \\
\mathrm{MIC}_{90} \\
>200\end{array}$ & $\begin{array}{l}\text { Range } \\
0.5-2 \\
\text { MIC }_{90} \\
\text { N/A }\end{array}$ & $\begin{array}{l}\text { Range } \\
<0.5-100 \\
\text { MIC }_{90} 16\end{array}$ \\
\hline Gentamicin & $\begin{array}{l}\text { Range } \\
5-20 \\
\mathrm{MIC}_{90} \\
20\end{array}$ & $\begin{array}{l}\text { Range } \\
5-20 \\
\text { MIC }_{90} 20\end{array}$ & $\begin{array}{l}\text { Range } \\
10-20 \\
\text { MIC }_{90} \\
\text { N/A }\end{array}$ & $\begin{array}{l}\text { Range } \\
10->200 \\
\mathrm{MIC}_{90} \\
>200\end{array}$ & $\begin{array}{l}\text { Range } \\
5-10 \\
\mathrm{MIC}_{90} \\
\text { N/A }\end{array}$ & $\begin{array}{l}\text { Range } \\
2.5-20 \\
\text { MIC }_{90} 10\end{array}$ \\
\hline Imipenem & $\begin{array}{l}\text { Range } \\
<0.625- \\
20 \\
\text { MIC }_{90} 5\end{array}$ & $\begin{array}{l}\text { Range } \\
<0.625-5 \\
\text { MIC }_{90} 5\end{array}$ & $\begin{array}{l}\text { Range } \\
1.25-20 \\
\text { MIC }_{90} \\
\text { N/A }\end{array}$ & $\begin{array}{l}\text { Range } \\
1.25->200 \\
\mathrm{MIC}_{90} \\
>200\end{array}$ & $\begin{array}{l}\text { Range } \\
<0.625- \\
1.25 \\
\mathrm{MIC}_{90} \\
\text { N/A }\end{array}$ & $\begin{array}{l}\text { Range } \\
0.625-20 \\
\text { MIC }_{90} 10\end{array}$ \\
\hline
\end{tabular}

Cef-S, cefovecin-susceptible based on disc diffusion; Cef-R, cefovecin-resistant based on disc diffusion; N/A, not applicable 